\title{
Plasma Levels of Myonectin But Not Myostatin or Fibroblast-Derived Growth Factor 21 Are Associated with Insulin Resistance in Adult Humans without Diabetes Mellitus
}

Freddy J. K. Toloza', Jose O. Mantilla-Rivas ${ }^{1}$, Maria C. Pérez-Matos', Maria L. RicardoSilgado', Martha C. Morales-Alvarez', Jairo A. Pinzón-Cortés', Maritza Pérez-Mayorga², Martha L. Arévalo-Garcia ${ }^{3}$, Giovanni Tolosa-González ${ }^{3}$ and Carlos O. Mendivil ${ }^{1,3 *}$

'Diabetes, Lipids and Metabolism Laboratory, School of Medicine, Universidad de los Andes, Bogotá, Colombia, ${ }^{2}$ Molecular Epidemiology of Endocrine Diseases Group, School of Medicine, Universidad Militar Nueva Granada, Bogotá, Colombia, ${ }^{3}$ Section of Endocrinology, Fundación Santa Fe de Bogotá, Bogotá, Colombia

OPEN ACCESS

Edited by:

Jan Polák,

Charles University, Czechia

Reviewed by: Amaia Rodríguez, Universidad de Navarra, Spain

Carlos A. Aguilar-Salinas, Instituto Nacional de Ciencias Medicas y Nutricion, Mexico

*Correspondence:

Carlos O. Mendivil cmendivi@uniandes.edu.co, carlosolimpo@gmail.com

Specialty section: This article was submitted to Diabetes,

a section of the journa

Frontiers in Endocrinology

Received: 22 September 2017 Accepted: 05 January 2018 Published: 31 January 2018

Citation:

Toloza FJK, Mantilla-Rivas JO, Pérez-Matos MC, RicardoSilgado ML, Morales-Alvarez MC, Pinzón-Cortés JA, Pérez-Mayorga M,

Arévalo-Garcia ML, TolosaGonzález G and Mendivil CO (2018) Plasma Levels of Myonectin But Not Myostatin or Fibroblast-Derived

Growth Factor 21 Are Associated with Insulin Resistance in Adult Humans without Diabetes Mellitus.

Front. Endocrinol. 9:5.

doi: 10.3389/fendo.2018.00005
Background: Myokines are a group of protein mediators produced by skeletal muscle under stress or physical exertion. Even though their discovery and effects in cell culture and animal models of disease have elicited great enthusiasm, very little is known about their role in human metabolism. We assessed whether plasma concentrations of three known myokines [myonectin, myostatin, and fibroblast-derived growth factor 21 (FGF21)] would be associated with direct and indirect indicators of insulin resistance (IR) in individuals who did not have a diagnosis of diabetes.

Methods: We studied 81 adults of both sexes comprising a wide range of body adiposity and insulin sensitivity. All participants underwent a thorough clinical assessment and a 5-point oral glucose tolerance test with calculation of multiple IR and insulin sensitivity indices. Twenty-one of them additionally underwent a hyperinsulinemic-euglycemic clamp with determination of steady-state whole-body insulin-stimulated glucose disposal ("M"). We compared plasma myokine concentrations across quartiles of IR indices and clinical IR surrogates, and explored the correlation of each myokine with the $M$-value.

Results: Plasma myonectin levels increased monotonically across quartiles of the incremental area under the insulin curve (higher values indicate more $I R)(p$-trend $=0.021)$ and decreased monotonically across quartiles of the insulin sensitivity index (ISI - higher values indicate less IR) ( $p$-trend $=0.012$ ). After multivariate adjustment for other relevant determinants of IR (body mass index, age, and sex), the negative association of myonectin with ISI persisted (standardized beta $=-0.235, p=0.023$ ). Myostatin was not associated with any clinical IR indicator or direct IR index measure. In multivariate analyses, FGF-21 showed a trend toward a positive correlation with glucose disposal that did not reach statistical significance (standardized beta $=0.476, p=0.091$ ).

Conclusion: The secretion of myonectin may constitute an attempt at a compensatory mechanism against IR in humans.

Keywords: myokine, myostatin, myonectin, fibroblast-derived growth factor 21, insulin resistance, obesity, muscle, diabetes mellitus 


\section{INTRODUCTION}

Insulin resistance (IR) is a key pathogenic mechanism for type 2 diabetes (DM2) and multiple other diseases. Recent evidence suggests that skeletal muscle is not only a target of insulin action but also a relevant endocrine organ as well. When muscle tissue is under stress it responds by secreting myokines, proteins with the ability to influence inflammation, glucose disposal, and adipose tissue phenotype via paracrine, autocrine, and possibly endocrine signaling (1). Despite their great potential as key metabolic players, our understanding of the role of myokines on human physiology and disease is still extremely limited. We aimed to study the association of circulating levels of three myokines: myostatin, myonectin, and fibrobast-derived growth factor 21 (FGF-21), with objective measures of IR in adult humans.

Myostatin was discovered in 1997 after a targeted search for proteins of the transforming growth factor superfamily (2). Myostatin is a negative regulator of the differentiation of myoblasts to myotubes in vitro (3), and myotubes from obese women secrete more myostatin than those of lean counterparts (4). Studies in murine models have found less inflammation and obesity-induced IR in myostatin knockout animals (5). Furthermore, antibodies against myostatin prevented age-induced sarcopenia in mice (6). However, the evidence about the influence of myostatin on insulin action and IR seems contradictory: Plasma myostatin decreases with increasing number of metabolic syndrome criteria in human patients (7). The relationship between myostatin and insulin action in humans is largely unknown.

Myonectin (also called CTRP15-C1q/TNF-related protein 15) was discovered in 2011 by Seldin et al. (8). Myonectin was shown to improve fatty acid (FA) uptake by cultured hepatocytes (8) and to increase in plasma of women after aerobic exercise training (9). Of note, during early stages of research, another protein (CTRP5) was also named myonectin. However, since CTRP15 is more selectively secreted by muscle tissue, the name myonectin was assigned to CTRP15 (8). Very little is known about the impact of myonectin on whole-body insulin action in humans.

Fibroblast growth factor 21 (FGF-21) is a pleiotropic hormone secreted mostly by the liver (10) but also by muscle under stress (11). FGF-21 activates brown adipose tissue thermogenesis in humans (12). FGF-21 levels correlate positively with body adiposity in humans (13), and are paradoxically higher in patients with the metabolic syndrome (14). Plasma FGF-21 levels increase in obese subjects and correlate positively with homeostasis model assessment of insulin resistance (HOMA-IR) (15). Additionally, when obese subjects experience weight loss with dietary or surgical intervention, FGF-21 levels decrease and IR-associated features improve (16). Preliminary human trials have shown positive effects of FGF-21 administration on metabolic parameters (17). These apparently contradicting findings make it relevant to understand the association of FGF-21 with directly measured IR in humans.

Given the potential but still unknown influence of myokines on nutrient and energy metabolism, we aimed to evaluate the association of circulating levels of myostatin, myonectin, and FGF-21 with whole-body IR in individuals without known diabetes mellitus. We also explored the association of these myokines with IR-associated features in our study participants.

\section{MATERIALS AND METHODS}

\section{Study Subjects}

We studied 81 adults of both sexes, aged 30-69, residents of Bogotá, Colombia, who had no prior diagnosis of diabetes mellitus. Sampling was not probabilistic and was done by open convocation through various channels (email, posters, referred by other participants). However, we intentionally included patients with a wide range of body mass indexes (BMIs) and presumably different IR status. Besides known diabetes, other exclusion criteria were pregnancy, use of anti-diabetic medications, endocrine diseases, and use of anticoagulants or metformin for any indication. We also excluded patients who were acutely ill, or whose plasma high-sensitivity C-reactive protein (hsCRP) was above $10 \mathrm{mg} / \mathrm{L}$. All subjects provided written informed consent.

We estimated the study's power with the expression for crosssectional studies that estimate a linear correlation coefficient (18). Given our study sample, the probability of finding a true linear correlation of at least 0.3 between the insulin sensitivity index by Gutt (ISI-Gutt) and each myokine under a 5\% probability of type I error was $82 \%$ for myonectin, $84.5 \%$ for myostatin, and $83 \%$ for FGF-21.

The study was approved by the Internal Review Board (Comité de Ética) of Universidad de los Andes according to minute 307 of 2014. The study complied with scientific, technical and administrative norms for health research dictated by resolution 008430 - 1993 of the Colombian Ministry of Health and with the principles stated by the Declaration of Helsinki. All study subjects underwent an informed consent procedure and provided written informed consent.

\section{Clinical Assessment, Oral Glucose Tolerance Test (OGTT), and Hyperinsulinemic Clamp}

We measured in all participants resting blood pressure, height, weight and abdominal circumference. Total and segmental percent body fat, percent lean mass, absolute lean mass (kg), and percent abdominal fat were determined with a tetrapolar biological impedance meter $\left(\mathrm{BC}_{4} 45\right.$, TANITA $\left.^{\circledR}\right)$. This device estimates lean body mass (LBM) by measuring electrical impedance across two pairs of electrodes (hands and feet soles) and then inputting the measured impedance plus the gender, age and height into patented equations developed and validated in several thousand participants of different ethnicities, to predict percent LBM. Fasting blood samples were obtained in potassium oxalate tubes for measurement of fasting glucose and in EDTA tubes for all other determinations including myokines. After prompt plasma separation, a protease inhibitor cocktail was added and total plasma was separated in aliquots for each myokine and frozen at $-80^{\circ} \mathrm{C}$ until analyzed.

For the OGTT patients arrived after 8-12 h overnight fast, and received a load of $75 \mathrm{~g}$ of glucose diluted in $300 \mathrm{~mL}$ of water 
to be consumed in less than 5 min. Blood samples were drawn for the measurement of plasma glucose and insulin measurement at times $0,30,60,90$, and 120 postglucose challenge. Patients could not smoke, ingest food or do significant physical activity during the OGTT. We selected completely at random a study subsample of 21 participants who additionally underwent a hyperinsulinemic-euglycemic clamp (19). After an overnight fast, subjects were admitted to a clinical research center, where two IV catheters were placed in the antecubital area of both arms for insulin and dextrose infusion. Another catheter was placed in the dorsal area of the hand ipsilateral to the dextrose infusion for blood sampling. The hand for capillary blood sampling and glucose measurement was placed inside a heated-hand box (The University of Vermont Medical, USA). This device allows the maintenance of a constant controlled temperature $\left(56-58^{\circ} \mathrm{C}\right)$ in order to arterialize venous blood. A short-acting human insulin analog (Humalog, Lilly) was infused at an initial priming rate of $100 \mathrm{mU} / \mathrm{m}^{2} / \mathrm{min}$ that was reduced to $90 \mathrm{mU} / \mathrm{m}^{2} / \mathrm{min}$ after $2 \mathrm{~min}$ and then in $20 \mathrm{mU} / \mathrm{m}^{2} / \mathrm{min}$ steps every $2 \mathrm{~min}$ until a dose of $40 \mathrm{mU} / \mathrm{m}^{2} / \mathrm{min}$ was reached. This rate was then kept constant for the next $110 \mathrm{~min}$, the whole procedure lasted $120 \mathrm{~min}$. A variable infusion of $20 \%$ glucose was started at the fourth minute and adjusted every $5 \mathrm{~min}$ in order to maintain the arterialized venous glucose concentration at $100 \mathrm{mg} / \mathrm{dL}(95-105 \mathrm{mg} / \mathrm{dL})$. Plasma glucose was measured by the glucose oxidase method using Accu-Chek Performa glucose meters (Roche, Germany). The main result of the clamp was whole-body insulin-stimulated glucose disposal at steady state ( $M$-value) (mg glucose $/ \mathrm{kg}$ body weight/min).

\section{Biochemical Measurements}

Fasting plasma glucose, plasma lipids, and creatinine were measured with enzymatic-colorimetric assays (Biosystems, Spain). Glycated hemoglobin Alc (HbAlc) was determined using a National Glycohemoglobin Standardization Programcertified boronate affinity technique (NycoCard ${ }^{\circledR}$ Reader II, Alere Technologies, Norway).

Myonectin was measured with the Aviscera Bioscience Myonectin ELISA (Cat\# SK00393-10, dynamic range 8-5,000 ng/ $\mathrm{mL}$, intra-assay CV: 6.0-8.0\%, interassay CV: $12.0-14.0 \%$ ), myostatin with the R\&D systems GDF-8/Myostatin Quantikine ELISA (Cat\#DGDF80, dynamic range 31.3-2,000 pg/mL, intra-assay CV: $1.8-5.4 \%$, interassay CV: $3.1-6.0 \%$ ) and FGF21 with the R\&D Systems Human FGF-21 Quantikine ELISA (Cat \#DF2100, dynamic range 31.3-2,000 pg/mL, intra-assay CV: $2.9-3.9 \%$, interassay CV: $5.2-10.9 \%)$. hsCRP was measured using R\&D Systems Human C-Reactive Protein/CRP Immunoassay (CAT\# DCRP00, dynamic range 0.010-50 ng/ $\mathrm{mL}$, intra-assay $\mathrm{CV}: 3.8-8.3 \%$, interassay CV: 6.0-7.0\%). All biomarker immunoassays were based on double-sandwich or competitive-ELISA techniques. Absorbance determinations were done in a BioTek Synergy HT Reader ${ }^{\circledR}$ using the appropriate wavelength according to protocol specifications. All measurements were performed in duplicate. Procedures were executed at the Diabetes, Lipids and Metabolism laboratory of Universidad de Los Andes, following current institutional biosafety protocols.

\section{Statistical Analyses}

We log-transformed plasma triglycerides before statistical analyses and estimated glomerular filtration rate using the Modified Diet for Renal Disease equation (20). Using plasma glucose and insulin values from the 5-point OGTT, we calculated IR indices based on fasting values: HOMA-IR (higher values indicate more IR) (21), quantitative insulin sensitivity check index (QUICKI, higher values indicate less IR) (22); IR indices based on postglucose challenge values: Incremental area under the insulin curve (iAUCins, higher values numbers indicate more IR), ISIGutt (higher values numbers indicate less IR) $(23,24)$. These are the mathematical expressions of the IR indices:

\section{- HOMA-IR:}

HOMA-IR: [FPI $(\mathrm{microU} / \mathrm{mL}) \times$ FPG $(\mathrm{mmol} / \mathrm{L})] / 22.5$.

- Insulin sensitivity index by Gutt (ISI):

ISI: $(\{75,000+[\mathrm{FPG}(\mathrm{mg} / \mathrm{L})-120 \mathrm{~min}$ glucose $(\mathrm{mg} / \mathrm{L})]\} \times 0.19$ $\times$ body weight $(\mathrm{kg})) / 120 \mathrm{~min} /[$ Mean plasma glucose $(\mathrm{mg} / \mathrm{L})]$

$/[\log$ mean serum insulin $(\mathrm{microU} / \mathrm{mL})]$.

- Corrected insulin response at $30 \mathrm{~min}$ (CIR-30):

CIR-30: $(100 \times 30 \mathrm{~min}$ insulin $) /\{30 \mathrm{~min}$ glucose $(\mathrm{mg} / \mathrm{dL})$

$\times[30 \mathrm{~min}$ glucose $(\mathrm{mg} / \mathrm{dL})-70(\mathrm{mg} / \mathrm{dL})]\}$.

- QUICKI:

QUICKI: 1/(Log fasting glucose [mmol/L]

+ Log fasting insulin $[\mu \mathrm{U} / \mathrm{mL}]$ ).

- iAUCins, calculated by the trapezoid method (23).

Comparisons of numerical variables between groups were made with Student's $t$-tests, after verification of normality and homosedasticity. Comparisons of categorical variables between groups were made with chi-square tests. Participants were placed in quartiles of IR indices and the mean concentration of each myokine was calculated for each quartile. Reported $p$-values for trend correspond to those associated with the coefficient of the slope in a linear regression in which mean values in each of the IR index quartiles were the independent variables, and mean concentrations of each myokine in each quartile were the dependent variable. The correlations between IR indices and plasma myokines, and between clinical variables and plasma myokines levels were examined using Spearman's correlation coefficients. All statistical tests were two-tailed at a significance level of 0.05. Analyses were performed with Statistical Package for Social Sciences version 23.0 (SPSS Inc., Chicago, IL, USA).

\section{RESULTS}

The study sample comprised 81 participants (57\% female). Mean age was 51.4 years, BMI $27.6 \mathrm{~kg} / \mathrm{m}^{2}$ for men and $25.8 \mathrm{~kg} / \mathrm{m}^{2}$ for women, abdominal circumference $95.1 \mathrm{~cm}$ for men and $82.5 \mathrm{~cm}$ for women. Mean systolic blood pressure (SBP), total, LDL, and 
HDL cholesterol, creatinine, and estimated glomerular filtration rate were within normal ranges (Table 1). Plasma myonectin did not differ significantly by sex. Plasma myostatin and FGF-21 were significantly higher in men $(p=0.017$ for myostatin, $p=0.044$ for FGF-21). However, these differences might only reflect a different muscle mass in men versus women. When plasma concentrations of myokines were adjusted by LBM ( $\mathrm{ng} / \mathrm{mL}$ of myokine per $\mathrm{kg}$ of LBM), only myonectin concentrations were different between sexes: women had significantly higher myonectin per $\mathrm{kg}$ of LBM than men (4.37 ng/mL/ $\mathrm{kg} \mathrm{LBM}$ versus $3.15 \mathrm{ng} / \mathrm{mL} / \mathrm{kg} \mathrm{LBM}$, $p=0.004)$. Among the OGTT-derived indices, ISI-Gutt exhibited the highest correlation with clamp-derived glucose disposal $(r=0.52, p=0.023)$. ISI-Gutt was also the only OGTT-derived significantly correlated with all other IR indices.

\section{Correlation between Myokines and Clinical Variables}

Plasma myokines were significantly correlated with each other (for myonectin and FGF-21 $r=0.35, p=0.003$; for myonectin and myostatin $p=0.38, p=0.001$; for FGF-21 and myostatin $r=0.36$, $p=0.001)$. Myonectin did not correlate significantly with any clinical IR feature. Myostatin showed a negative correlation with

TABLE 1 | Characteristics of study participants.

\begin{tabular}{|c|c|}
\hline & Total $(n=81)$ \\
\hline Age (years) & $51.4 \pm 10.1$ \\
\hline Weight (kg) & $70.4 \pm 13.8$ \\
\hline Height (cm) & $161.9 \pm 8.9$ \\
\hline Body mass index $\left(\mathrm{kg} / \mathrm{m}^{2}\right)$ & $26.7 \pm 4.1$ \\
\hline Body water percent (\%) & $49.8 \pm 4.8$ \\
\hline Body fat percent (\%) & $31.1 \pm 7.3$ \\
\hline Visceral fat percent (\%) & $8.9 \pm 3.8$ \\
\hline Lean mass percent (\%) & $65.9 \pm 7.2$ \\
\hline Bone mass (kg) & $2.4 \pm 0.5$ \\
\hline Abdominal circumference (cm) & $88.2 \pm 12.7$ \\
\hline Systolic blood pressure (mmHg) & $118 \pm 15.4$ \\
\hline Diastolic blood pressure $(\mathrm{mmHg})$ & $71.4 \pm 17.3$ \\
\hline Fasting glucose (mmol/L) & $5.32 \pm 0.54$ \\
\hline Glycated hemoglobin (HbA1c) (\%) & $5.5 \pm 1.4$ \\
\hline Creatinine $(\mu \mathrm{mol} / \mathrm{L})$ & $71.6 \pm 15.0$ \\
\hline Total cholesterol (mmol/L) & $5.17 \pm 1.27$ \\
\hline Triglycerides (mmol/L) & $1.76 \pm 0.88$ \\
\hline HDL cholesterol (mmol/L) & $1.14 \pm 0.32$ \\
\hline LDL cholesterol (mmol/L) & $3.26 \pm 1.18$ \\
\hline C-reactive protein (mmol/L) & $24.2 \pm 9.5$ \\
\hline HOMA-IR & $4.51 \pm 4.41$ \\
\hline $\log (\mathrm{HOMA}-\mathrm{IR})$ & $0.51 \pm 0.33$ \\
\hline HOMA-B & $226.9 \pm 253.4$ \\
\hline iAUCins ( $\mu \cup I \mathrm{~min} / \mathrm{mL})$ & $4,852 \pm 5,850$ \\
\hline QUICKI & $0.324 \pm 0.035$ \\
\hline $\mathrm{M}$ value (mg/kg body weight/min) & $5.20 \pm 1.88$ \\
\hline Myonectin (ng/mL) & $170.0 \pm 67.8$ \\
\hline FGF-21 (pg/mL) & $185.3 \pm 128.4$ \\
\hline Myostatin (pg/mL) & $2,963 \pm 1,878$ \\
\hline
\end{tabular}

Data are mean \pm SD unless indicated otherwise. M-value: whole-body steady-state glucose disposal in the hyperinsulinemic-euglycemic clamp.

HOMA-IR, homeostasis model assessment of insulin resistance; HOMA-B, homeostasis model assessment-beta cell; QUICKI, quantitative insulin sensitivity check index; iAUCins, incremental area under the insulin curve; FGF-21, fibroblast grow factor. body fat percent $(r=-0.36, \mathrm{p}=0.001)$, and a positive correlation with LBM percent $(r=0.35, p=0.001)$ (Table 2$)$. Similarly, FGF21 showed a positive correlation with LBM percent $(r=0.37$, $p=0.001)$.

\section{Plasma Myokines and IR/Insulin Secretion}

Characteristics of the random subsample of patients who underwent the hyperinsulinemic-euglycemic clamp did not differ significantly from those of the complete study sample (Table 3).

Plasma myonectin was positively associated with IR. The univariate Spearman linear correlation coefficient between myonectin and ISI was $r=-0.12(p=0.29)$, but between myonectin and iAUCins it was $r=0.24(p=0.04)$. Myonectin levels increased linearly across quartiles of the iAUCins (Q1: $155 \mathrm{ng} / \mathrm{mL}, \mathrm{Q} 4$ : $179 \mathrm{ng} / \mathrm{mL}$, $p$-trend $=0.021)$, and decreased linearly across quartiles of the ISI (Q1: $178 \mathrm{ng} / \mathrm{mL}, \mathrm{Q} 4: 150 \mathrm{ng} / \mathrm{mL}, p$-trend $=0.012$ ) (Figure 1). Unexpectedly, myonectin also showed a univariate positive correlation with $\mathrm{M}$ value in the hyperinsulinemiceuglycemic clamp $(r=0.50, p=0.034)$ (Table 4; Figure 2). However, after adjustment for key determinants of IR (age, sex, and BMI) in multiple linear regression models, only the negative association of myonectin with the ISI persisted [normalized coefficient (nBeta) $=-0.235, p=0.023$, while the associations with iAUCins and insulin-stimulated glucose uptake disappeared ( $p=0.30$ and $p=0.11$, respectively). When percent body fat replaced BMI as a measure of body adiposity in multivariate models, the negative association of myonectin with the ISI was not modified (nBeta $=-0.240, p=0.024$ ).

The univariate Spearman's correlation coefficient between myostatin and ISI was $r=-0.06(p=0.61)$, and between myonectin and iAUCins it was $r=0.13(p=0.25)$. Myostatin did not change systematically across quartiles of any of the indices reflecting IR, but trended toward a positive association with the iAUCins in the multivariate-adjusted model (standardized beta $=-0.206, p=0.072$ ).

TABLE 2 | Spearman's correlation coefficients between plasma myokines and clinical surrogates of insulin resistance.

\begin{tabular}{lrccccl}
\hline & \multicolumn{2}{c}{ FGF-21 } & \multicolumn{2}{c}{ Myonectin } & \multicolumn{2}{c}{ Myostatin } \\
\cline { 2 - 7 } & \multicolumn{1}{c}{$\boldsymbol{r}$} & $\boldsymbol{p}$ & $\boldsymbol{r}$ & $\boldsymbol{p}$ & $\boldsymbol{r}$ & $\boldsymbol{p}$ \\
\hline BMl & -0.03 & 0.78 & -0.10 & 0.42 & -0.028 & 0.80 \\
Body fat percent & -0.32 & 0.005 & -0.08 & 0.52 & -0.36 & $0.001^{\mathrm{a}}$ \\
Abdominal fat percent & -0.06 & 0.59 & 0.01 & 0.92 & 0.120 & 0.29 \\
Lean body mass & 0.37 & $0.001^{\mathrm{a}}$ & 0.14 & 0.22 & 0.35 & $0.001^{\mathrm{a}}$ \\
percent & & & & & & \\
Waist circumference & 0.07 & 0.55 & -0.10 & 0.42 & 0.013 & 0.91 \\
Systolic blood pressure & 0.00 & 0.98 & -0.08 & 0.52 & -0.104 & 0.36 \\
Fasting plasma glucose & -0.01 & 0.93 & -0.11 & 0.34 & -0.064 & 0.58 \\
HbA1c & 0.19 & 0.13 & 0.15 & 0.25 & 0.35 & 0.004 \\
Log (triglycerides) & 0.00 & 0.99 & -0.05 & 0.68 & 0.030 & 0.79 \\
HDL cholesterol & -0.05 & 0.69 & -0.03 & 0.81 & -0.076 & 0.50 \\
LDL cholesterol & -0.05 & 0.65 & -0.03 & 0.82 & 0.030 & 0.79 \\
hsCRP & -0.13 & 0.25 & 0.01 & 0.92 & -0.095 & 0.40 \\
Fasting insulinemia & -0.13 & 0.28 & -0.01 & 0.92 & 0.142 & 0.21
\end{tabular}

a Significant at a Bonferroni-adjusted significance level of 0.00384 $B M l$, body mass index; hsCRP, high sensitivity $C$-reactive protein. 
Fibroblast-derived growth factor 21 changed across quartiles of the iAUCins $(\mathrm{Q} 1=169.3, \mathrm{Q} 4=240.6)$, but this trend did not reach statistical significance $(p$-trend $=0.16)$. Similar to

TABLE 3 | Comparison of demographic, clinical, and laboratory characteristics of study participants who underwent or did not undergo the hyperinsulinemiceuglycemic clamp.

\begin{tabular}{|c|c|c|c|}
\hline & Clamp $(n=21)$ & No clamp $(n=60)$ & $p$ \\
\hline Age & $52.6 \pm 7.48$ & $51.61 \pm 11.12$ & 0.654 \\
\hline Weight & $69.97 \pm 10.38$ & $70.06 \pm 15.21$ & 0.977 \\
\hline Height & $162.74 \pm 9.35$ & $161.66 \pm 8.75$ & 0.652 \\
\hline BMI & $26.32 \pm 2.3$ & $26.69 \pm 4.76$ & 0.648 \\
\hline$\%$ body water & $49.75 \pm 4.39$ & $49.8 \pm 4.98$ & 0.964 \\
\hline$\%$ body fat & $31.37 \pm 7.04$ & $30.98 \pm 7.34$ & 0.836 \\
\hline$\%$ abdominal fat & $8.62 \pm 2.89$ & $8.95 \pm 4.13$ & 0.694 \\
\hline Lean mass (kg) & $46.31 \pm 9.09$ & $45.78 \pm 9.74$ & 0.823 \\
\hline Lean mass (\%) & $66.1 \pm 6.45$ & $65.82 \pm 7.43$ & 0.908 \\
\hline Bone mass & $2.44 \pm 0.46$ & $2.82 \pm 3.08$ & 0.358 \\
\hline Abdominal girth & $86.21 \pm 8.12$ & $88.86 \pm 13.89$ & 0.326 \\
\hline Systolic BP & $115.9 \pm 11.48$ & $118.68 \pm 16.52$ & 0.418 \\
\hline Diastolic BP & $74.65 \pm 9.75$ & $74.47 \pm 11.1$ & 0.948 \\
\hline $\begin{array}{l}\text { Fasting capillary glucose } \\
(\mathrm{mmol} / \mathrm{L})\end{array}$ & $5.30 \pm 0.39$ & $5.34 \pm 0.59$ & 0.772 \\
\hline $\mathrm{HbA} 1 \mathrm{c}(\%)$ & $5.37 \pm 0.69$ & $5.73 \pm 1.49$ & 0.180 \\
\hline Creatinine ( $\mu \mathrm{mol} / \mathrm{L})$ & $73.5 \pm 14.2$ & $71.7 \pm 15.0$ & 0.621 \\
\hline Total cholesterol (mmol/L) & $5.03 \pm 1.33$ & $5.18 \pm 1.23$ & 0.664 \\
\hline Log(triglycerides) & $2.17 \pm 1.87$ & $2.20 \pm 1.89$ & 0.568 \\
\hline HDLc (mmol/L) & $1.06 \pm 0.27$ & $1.16 \pm 0.33$ & 0.180 \\
\hline LDLc (mmol/L) & $3.24 \pm 1.19$ & $3.23 \pm 1.16$ & 0.972 \\
\hline C-reactive protein (mmol/L) & $25.1 \pm 13.5$ & $31.1 \pm 45.1$ & 0.462 \\
\hline Fasting glucose (mmol/L) & $5.30 \pm 0.38$ & $5.36 \pm 0.58$ & 0.634 \\
\hline Glucose at $30 \mathrm{~min}(\mathrm{mmol} / \mathrm{L})$ & $7.48 \pm 1.20$ & $7.45 \pm 2.1$ & 0.953 \\
\hline Glucose at $60 \mathrm{~min}(\mathrm{mmol} / \mathrm{L})$ & $7.53 \pm 1.69$ & $7.86 \pm 2.73$ & 0.517 \\
\hline Glucose at $90 \mathrm{~min}(\mathrm{mmol} / \mathrm{L})$ & $6.46 \pm 1.54$ & $7.20 \pm 2.63$ & 0.130 \\
\hline Glucose at $120 \mathrm{~min}(\mathrm{mmol} / \mathrm{L})$ & $6.14 \pm 1.10$ & $6.59 \pm 2.57$ & 0.280 \\
\hline Basal insulinemia (pmol/L) & $167.5 \pm 166.1$ & $118.7 \pm 107.2$ & 0.229 \\
\hline Insulinemia at $30 \mathrm{~min}(\mathrm{pmol} / \mathrm{L})$ & $472.5 \pm 451.1$ & $503.3 \pm 749.2$ & 0.827 \\
\hline Insulinemia at $60 \mathrm{~min}(\mathrm{pmol} / \mathrm{L})$ & $564.9 \pm 431.7$ & $502.7 \pm 509.9$ & 0.598 \\
\hline Insulinemia at $90 \mathrm{~min}(\mathrm{pmol} / \mathrm{L})$ & $419.9 \pm 341.4$ & $341.2 \pm 365.8$ & 0.388 \\
\hline HOMA-IR & $5.62 \pm 5.56$ & $4.1 \pm 3.87$ & 0.270 \\
\hline Log (Homa) & $0.59 \pm 0.35$ & $0.49 \pm 0.33$ & 0.263 \\
\hline HOMA-B & $310.31 \pm 397.49$ & $196.7 \pm 165.28$ & 0.227 \\
\hline ISI (Gutt) & $70.61 \pm 17.48$ & $74 \pm 22.49$ & 0.492 \\
\hline QUICKI & $0.32 \pm 0.03$ & $0.33 \pm 0.03$ & 0.240 \\
\hline ¡AUC-insulin & $5,207 \pm 4,840$ & $4,724 \pm 6,224$ & 0.723 \\
\hline
\end{tabular}

HOMA-IR, homeostasis model assessment of insulin resistance; HOMA-B, homeostasis model assessment-beta cell; QUICKI, quantitative insulin sensitivity check index; iAUCins, incremental area under the insulin curve; ISI-Gutt, insulin sensitivity index by Gutt; FGF-21, fibroblast grow factor; BMI, body mass index. myonectin, FGF-21 had a positive albeit non-significant correlation with glucose disposal in the clamp $(r=0.38, p=0.099)$ (Table 3), that was also evident in the adjusted linear model (standardized beta $=0.476, p=0.091$ ). The univariate Spearman's correlation coefficient between FGF-21 and ISI was $r=-0.02$ $(p=0.87)$, while between myonectin and iAUCins it was $r=0.16$ $(p=0.18)$.

\section{DISCUSSION}

In this study, we report the association between plasma concentrations of various myokines and objectively measured IR in humans. We found that despite their mutual correlation, myokines exhibit different associations with IR.

In our sample of subjects without known diabetes, myonectin concentrations were positively associated with measures of IR like the iAUCins and negatively associated with insulin sensitivity measures like the ISI-Gutt. Adjustment for major covariates rendered the association with iAUCins non-significant, but the negative association with ISI persisted. In mice, myonectin synthesis and secretion is induced by the hormonal response to the postabsorptive state. Myonectin is able to induce fatty acid uptake by liver and adipose tissue (8). A previous study in 28 women who performed aerobic exercise for 10 weeks identified a positive correlation between semi-quantitatively determined plasma myonectin (western blot) and HOMA-IR (9). Integration of our findings with this prior evidence suggests that myonectin may constitute a muscle-based nutrient-sensing mechanism that works to increase nutrient uptake in other tissues. Thus, increased myonectin levels in the presence of IR may represent an attempt at a compensatory mechanism.

Recombinant myonectin has shown to reduce the concentrations of FFA in mice (8), so it is conceivable that myonectininduced FFA uptake would reduce the deleterious effect of FFA on systemic insulin sensitivity. We did not measure FFA in our study participants, so we do not have enough information to either confirm or rule out this hypothesis, which would reinforce a role for myonectin as a compensatory mechanism against IR. Future studies of the impact of myonectin on IR in humans may benefit from simultaneous measurement of FFA.

Yet another possibility is that high body adiposity is a common precursor to both IR and increased myonectin. Obesity is
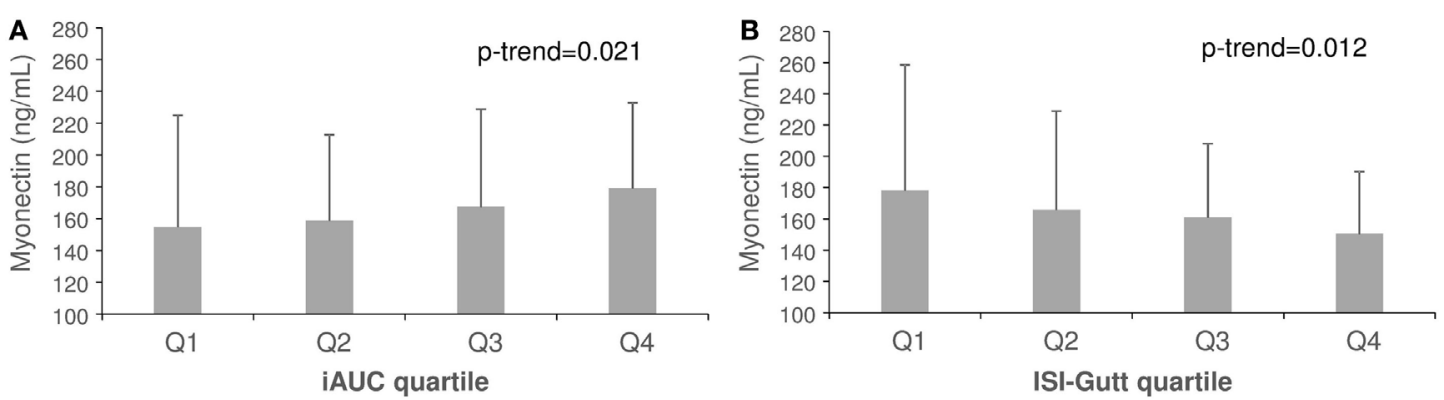

FIGURE 1 | Mean concentrations of myonectin across quartiles of incremental area under the insulin curve (A) and insulin sensitivity index (B). Error bars represent SDs. 
TABLE 4 | Mean values of plasma myokines across quartiles of insulin resistance and insulin secretion indices from the OGTT.

\begin{tabular}{|c|c|c|c|c|c|c|c|c|c|c|c|c|}
\hline & \multicolumn{3}{|c|}{ HOMA-IR } & \multicolumn{3}{|c|}{ ¡AUCins } & \multicolumn{3}{|c|}{ ISI-Gutt } & \multicolumn{3}{|c|}{ QUICKI } \\
\hline & Q1 & Q4 & $p$-Trend & Q1 & Q4 & $p$-Trend & Q1 & Q4 & $p$-Trend & Q1 & Q4 & $p$-Trend \\
\hline Myonectin (ng/mL) & 159 (48) & $180.6(80)$ & 0.16 & $155(70)$ & $179(54)$ & 0.021 & $179(81)$ & $150(40)$ & 0.012 & $179(82)$ & $159(48)$ & 0.15 \\
\hline Myostatin (pg/mL) & $\begin{array}{c}2,687 \\
(1,770)\end{array}$ & $3,631(2,154)$ & 0.49 & $\begin{array}{c}2,891 \\
(1,702)\end{array}$ & $\begin{array}{c}3,391 \\
(2,145)\end{array}$ & 0.42 & $\begin{array}{c}3,179 \\
(1,894)\end{array}$ & $\begin{array}{c}2,964 \\
(1,684)\end{array}$ & 0.53 & $\begin{array}{c}3,415 \\
(1,976)\end{array}$ & $\begin{array}{c}2,688 \\
(1,770)\end{array}$ & 0.57 \\
\hline FGF-21 (pg/mL) & $170(94)$ & 163.8 (103) & 0.82 & (169) (90) & 241 (193) & 0.16 & 180 (109) & 165 (93) & 0.62 & $166(106)$ & $170(94)$ & 0.85 \\
\hline
\end{tabular}

Data are mean (SD).

HOMA-IR, homeostasis model assessment of insulin resistance; QUICKI, quantitative insulin sensitivity check index; iAUCins, incremental area under the insulin curve; ISI-Gutt, insulin sensitivity index by Gutt.
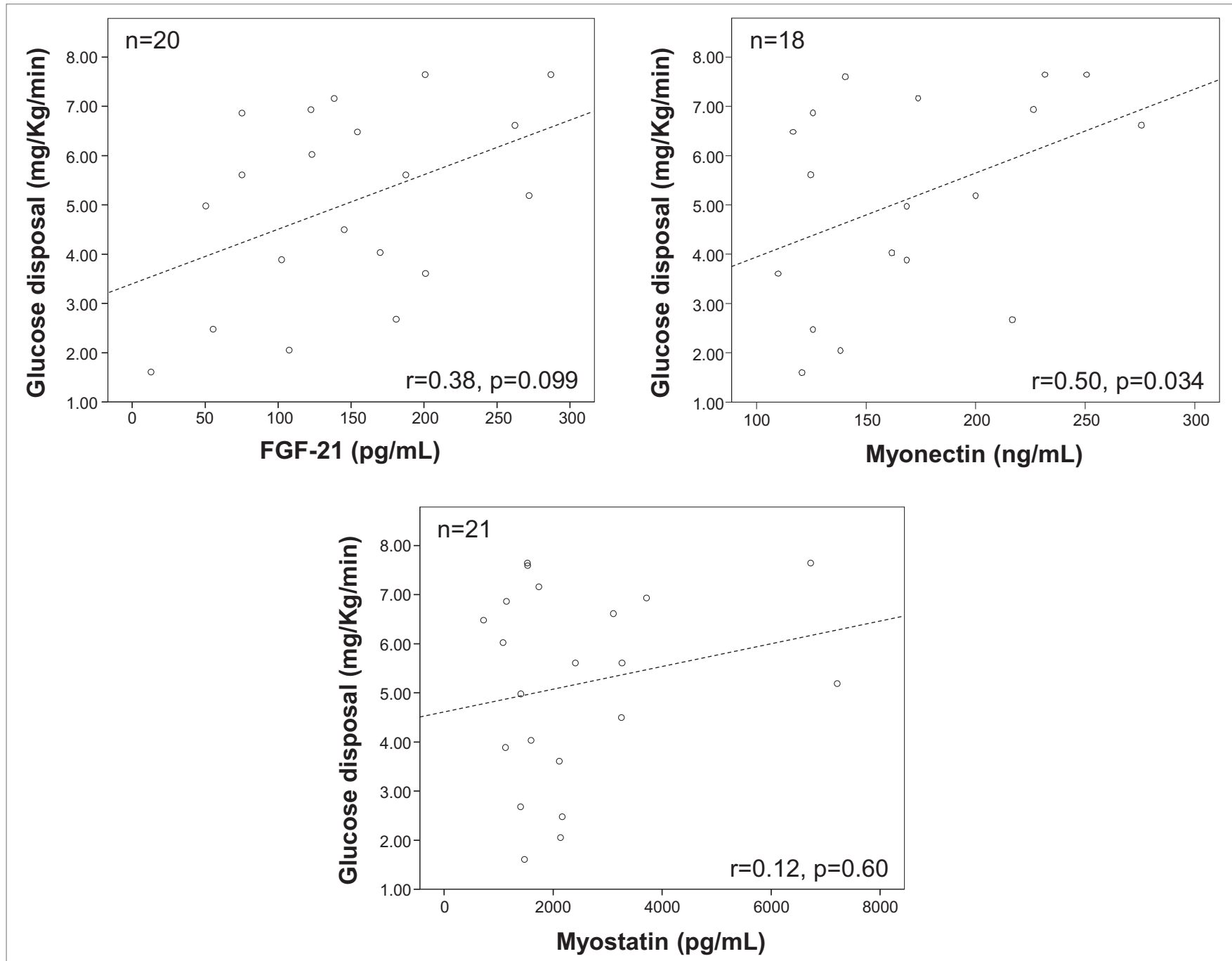

FIGURE 2 | Scatterplots of plasma myonectin concentrations versus whole body insulin-stimulated glucose disposal at steady state in the hyperinsulinemiceuglycemic clamp. $r$-Values are Spearman's correlation coefficients and their associated $p$-values.

characterized by hyperleptinemia, and leptin administration has demonstrated to increase transcription of the myonectin mRNA in mouse myocytes (25). However, the fact that the association between plasma myonectin and ISI was independent of BMI or percent body fat in multivariate models suggests that the link between myonectin and IR is at least partially independent of the leptin axis.

Myostatin on the other hand was not significantly correlated with IR. In a study of 10 obese individuals (26), a 6-month aerobic exercise intervention induced a reduction in both muscle 
and plasma myostatin and an improvement in IR measured by the intravenous glucose tolerance test-minimal model. In the same report, myostatin administration to mice directly induced IR (27). A study in humans with or without diabetes found an association between myostatin and the HOMA-IR, though only among participants without diabetes (27), and only at the gene expression level, not for the circulating protein. Contrastingly, plasma myostatin did not correlate with one-year weight loss among obese participants of a non-surgical weight loss program (28). Also against a direct involvement of myostatin in human IR, a study of 246 subjects found people with more severe metabolic syndrome to have lower, not higher plasma myostatin (7). Thus, evidence about the involvement of myostatin on human IR is conflicting. Compared to prior reports in younger individuals (mean age 20 years), our study subjects exhibited lower average plasma myostatin concentrations $(2.96 \mathrm{ng} / \mathrm{mL}$ in our study versus $12.3 \mathrm{ng} / \mathrm{mL}$ ) (29). In fact, plasma myostatin decreases with age (29), as it happens with muscle mass. A host of findings suggest that even though myostatin secretion is proportional to total muscle mass, its effect may constitute a negative-feedback signal that reduces differentiation of myotubes $(3,6)$. The apparent inconsistencies regarding the influence of myostatin on IR may be explained if myostatin action is mostly paracrine and thus not reflected by circulating concentrations.

Fibroblast-derived growth factor 21 levels correlated positively with percent LBM, in accordance with its action as a promoter of thermogenesis and "browning" of adipose tissue in animal models (30). Plasma FGF-21 exhibited a monotonic, yet not linearly significant association with the iAUCins. Despite this pattern, FGF-21 tended toward a positive association with glucose disposal in the clamp, suggesting a failed or overwhelmed compensatory mechanism. Prior evidence points in the same direction, as plasma FGF-21 correlates positively with adiposity, fasting insulin, and triglycerides in humans (14). Patients with diabetes have elevated FGF-21 levels (31), and plasma FGF-21 has been associated with IR measures including the AUCins in community-dwelling individuals (32). Another potential explanation for the increased FGF-21 in obesity and IR is the existence of a FGF-21-resistant state, a hypothesis that has been proven in a diet-induced obesity mouse model (33).

It is interesting that some, but not all myokines displayed a significant association with IR. Despite sharing a common tissue source, myokines possess very different structural and physiological properties. Thus, myostatin induces in muscle cells a set of effects opposite to those of myonectin $(3,6,8)$, and a similar pattern maybe expected at the whole-body level. In the case of FGF-21, high plasma levels and resistance to its effects have been documented in rodent models of obesity and diabetes $(11,33)$. It is conceivable that the secretion of FGF-21 as a compensatory hormone that increases energy expenditure (34), may occur only in the face of massive IR, while in individuals with a more moderate degree of IR it only manifests as the non-significant trend that we detected.

The main limitations of our study include its relatively small number of participants and the cross-sectional nature of the measurement of myokines and IR in a particular group of participants of Hispanic ethnicity, all of which limit the generalizability of our results. Another important limitation is that our study design does not allow us to infer the mechanism(s) explaining the observed association between myonectin and IR. However, our study is one small step closer to interventional studies in humans that may evaluate the direct influence of myokines on insulin action and metabolic disease.

In summary, we simultaneously studied three myokines in a sample of adult individuals without known diabetes and comprising a wide range of age and body adiposity. Our findings suggest that myonectin and probably FGF-21 constitute an attempt at a compensatory mechanism against IR. The association of myostatin with human IR is less clear and will require further study.

\section{ETHICS STATEMENT}

Full name of the ethics committee that approved the study: Comité de Ética Universidad de los Andes, minute 307 of 2014. Consent procedure used for human participants or for animal owners. All study subjects underwent an informed consent procedure and provided written informed consent. Any additional considerations of the study in cases where vulnerable populations were involved, for example minors, persons with disabilities or endangered animal species. Not applicable. The study complied with scientific, technical and administrative norms for health research dictated by resolution 008430 - 1993 of the Colombian Ministry of Health and with the principles stated by the Declaration of Helsinki.

\section{AUTHOR CONTRIBUTIONS}

FT contributed to study conception, execution, data collection, statistical analysis, and manuscript writing. JM-R contributed to study conception, execution, data collection, statistical analysis, and manuscript revision. MP-M contributed to study execution, data collection, statistical analysis, and manuscript revision. MR-S, MM-A, JP-C, MM, MA-G, and GT-G contributed to study execution, data collection, and manuscript revision. CM directed the study and contributed to study conception, execution, data collection, statistical analysis, and manuscript writing.

\section{ACKNOWLEDGMENTS}

We want to thank all study participants for their patience and willingness.

\section{FUNDING}

Funding for this study was provided by Departamento Administrativo de Ciencia, Tecnología e Investigación de Colombia, COLCIENCIAS. 


\section{REFERENCES}

1. Schnyder S, Handschin C. Skeletal muscle as an endocrine organ: PGC-1 $\alpha$, myokines and exercise. Bone (2015) 80:115-25. doi:10.1016/j.bone.2015.02.008

2. McPherron A, Lawler A, Lee S. Regulation of skeletal muscle mass in mice by a new TGF superfamily member. Nature (1997) 387:83-90. doi:10.1038/387083a0

3. Thomas M, Langley B, Berry C, Sharma M, Kirk S, Bass J, et al. Myostatin, a negative regulator of muscle growth, functions by inhibiting myoblast proliferation. J Biol Chem (2000) 275:40235-43. doi:10.1074/jbc.M004356200

4. Hittel D, Berggren J, Shearer J, Boyle K, Houmard J. Increased secretion and expression of myostatin in skeletal muscle from extremely obese women. Diabetes (2009) 58:30-8. doi:10.2337/db08-0943

5. Wilkes J, Lloyd D, Gekakis N. Loss-of-function mutation in myostatin reduces tumor necrosis factor production and protects liver against obesity-induced insulin resistance. Diabetes (2009) 58:1133-43. doi:10.2337/db08-0245

6. Camporez JP, Petersen MC, Abudukadier A, Moreira GV, Jurczak MJ, Friedman G, et al. Anti-myostatin antibody increases muscle mass and strength and improves insulin sensitivity in old mice. Proc Natl Acad Sci U S A (2016) 113:2212-7. doi:10.1073/pnas.1525795113

7. Han D, Chu-Su Y, Chiang C, Tseng F, Tseng P, Chen C, et al. Serum myostatin is reduced in individuals with metabolic syndrome. PLoS One (2014) 9:e108230. doi:10.1371/journal.pone.0108230

8. Seldin M, Peterson J, Byerly M, Wei Z, Wong G. Myonectin (CTRP15), a novel myokine that links skeletal muscle to systemic lipid homeostasis. J Biol Chem (2012) 287:11968-80. doi:10.1074/jbc.M111.336834

9. Lim S, Choi S, Koo B, Kang S, Yoon J, Jang H, et al. Effects of aerobic exercise training on $\mathrm{Clq}$ tumor necrosis factor $\alpha$-related protein isoform 5 (myonectin): association with insulin resistance and mitochondrial DNA density in women. J Clin Endocrinol Metab (2012) 97:E88-93. doi:10.1210/jc. 2011-1743

10. Nishimura T, Nakatake $\mathrm{Y}$, Konishi M, Itoh N. Identification of a novel FGF, FGF-21, preferentially expressed in the liver. Biochim Biophys Acta (2000) 1492:203-6. doi:10.1016/S0167-4781(00)00067-1

11. Fisher F, Maratos-Flier E. Understanding the physiology of FGF21. Annu Rev Physiol (2016) 78:223-41. doi:10.1146/annurev-physiol-021115-105339

12. Lee P, Linderman JD, Smith S, Brychta RJ, Wang J, Idelson C, et al. Irisin and FGF21 are cold-induced endocrine activators of brown fat function in humans. Cell Metab (2014) 19:302-9. doi:10.1016/j.cmet.2013.12.017

13. Giannini C, Feldstein AE, Santoro N, Kim G, Kursawe R, Pierpont B, et al. Circulating levels of FGF-21 in obese youth: associations with liver fat content and markers of liver damage. J Clin Endocrinol Metab (2013) 98:2993-3000. doi:10.1210/jc.2013-1250

14. Zhang X, Yeung D, Karpisek M, Stejskal D, Zhou Z, Liu F, et al. Serum FGF21 levels are increased in obesity and are independently associated with the metabolic syndrome in humans. Diabetes (2008) 57:1246-53. doi:10.2337/ $\mathrm{db} 07-1476$

15. Gallego-Escuredo JM, Gómez-Ambrosi J, Catalan V, Domingo P, Giralt M, Frühbeck G, et al. Opposite alterations in FGF21 and FGF19 levels and disturbed expression of the receptor machinery for endocrine FGFs in obese patients. Int J Obes (Lond) (2015) 39:121-9. doi:10.1038/ijo.2014.76

16. Gómez-Ambrosi J, Gallego-Escuredo JM, Catalán V, Rodríguez A, Domingo P, Moncada R, et al. FGF19 and FGF21 serum concentrations in human obesity and type 2 diabetes behave differently after diet- or surgically-induced weight loss. Clin Nutr (2017) 36:861-8. doi:10.1016/j.clnu.2016.04.027

17. Gaich G, Chien J, Fu H, Glass L, Deeg M, Holland W, et al. The Effects of LY2405319, an FGF21 analog, in obese human subjects with type 2 diabetes. Cell Metab (2013) 18:333-40. doi:10.1016/j.cmet.2013.08.005

18. Hulley SB, Cummings SR, Browner WS, Grady D, Newman TB. Designing Clinical Research: An Epidemiologic Approach. 4th ed. Philadelphia, PA: Lippincott Williams \& Wilkins (2013). 79 p. Appendix 6C.

19. deFronzo RA, Tobin JD, Andres R. Glucose clamp technique: a method for quantifying insulin secretion and resistance. Am J Physiol (1979) 237:E214-23.
20. Soares AA, Eyff TF, Campani RB, Ritter L, Camargo JL, Silveiro SP. Glomerular filtration rate measurement and prediction equations. Clin Chem Lab Med (2009) 47:1023-32. doi:10.1515/CCLM.2009.263

21. Matthews DR, Hosker JP, Rudenski AS, Naylor BA, Treacher DF, Turner RC. Homeostasis model assessment: insulin resistance and beta-cell function from fasting plasma glucose and insulin concentrations in man. Diabetologia (1985) 28:412-9. doi:10.1007/BF00280883

22. Katz A, Nambi SS, Mather K, Baron AD, Follmann DA, Sullivan G, et al. Quantitative insulin sensitivity check index: a simple, accurate method for assessing insulin sensitivity in humans. JClin Endocrinol Metab (2000) 85:2402-10. doi:10.1210/jcem.85.7.6661

23. Matthews J, Altman D, Campbell M, Royston P. Analysis of serial measurements in medical research. $\mathrm{Br}$ Med J (1990) 300:230-5. doi:10.1136/ bmj.300.6725.680-a

24. Gutt M, Davis CL, Spitzer SB, Llabre MM, Kumar M, Czarnecki EM, et al. Validation of the insulin sensitivity index (ISI $(0,120)$ ): comparison with other measures. Diabetes Res Clin Pract (2000) 47:177-84. doi:10.1016/ S0168-8227(99)00116-3

25. Rodríguez A, Becerril S, Méndez-Giménez L, Ramírez B, Sáinz N, Catalán $\mathrm{V}$, et al. Leptin administration activates irisin-induced myogenesis via nitric oxide-dependent mechanisms, but reduces its effect on subcutaneous fat browning in mice. Int J Obes (2015) 39:397-407. doi:10.1038/ijo.2014.166

26. Hittel D, Axelson M, Sarna N, Shearer J, Huffman K, Kraus W. Myostatin decreases with aerobic exercise and associates with insulin resistance. Med Sci Sports Exerc (2010) 42:2023-9. doi:10.1249/MSS.0b013e3181e0b9a8

27. Brandt C, Nielsen A, Fischer C, Hansen J, Pedersen B, Plomgaard P. Plasma and muscle myostatin in relation to type 2 diabetes. PLoS One (2012) 7:e37236. doi:10.1371/journal.pone.0037236

28. Tsioga M, Oikonomou D, Vittas S, Kalscheuer H, Roeder E, Wintgens K, et al. Plasma myostatin is only a weak predictor for weight maintenance in obese adults. Exp Clin Endocrinol Diabetes (2015) 123:466-72. doi:10.1055/s-00351559663

29. Han D, Huang C, Chen S, Yang W. Serum reference value of two potential doping candidates-myostatin and insulin-like growth factor-I in the healthy young male. J Int Soc Sports Nutr (2017) 14:2. doi:10.1186/s12970-016-0160-9

30. Kharitonenkov A, Shiyanova T, Koester A, Ford A, Micanovic R, Galbreath E, et al. FGF-21 as a novel metabolic regulator. J Clin Invest (2005) 115:1627-35. doi:10.1172/JCI23606

31. Cheng X, Zhu B, Jiang F, Fan H. Serum FGF-21 levels in type 2 diabetic patients. Endocr Res (2011) 36:142-8. doi:10.3109/07435800.2011.558550

32. Semba R, Sun K, Egan J, Crasto C, Carlson O, Ferrucci L. Relationship of serum fibroblast growth factor 21 with abnormal glucose metabolism and insulin resistance: the Baltimore Longitudinal Study of Aging. JClin Endocrinol Metab (2012) 97:1375-82. doi:10.1210/jc.2011-2823

33. Fisher F, Chui P, Antonellis P, Bina H, Kharitonenkov A, Flier J, et al. Obesity is a fibroblast growth factor 21 (FGF21)-resistant state. Diabetes (2010) 59:2781-9. doi:10.2337/db10-0193

34. Cuevas-Ramos D, Aguilar-Salinas CA. Modulation of energy balance by fibroblast growth factor 21. Horm Mol Biol Clin Investig (2016) 30. doi:10.1515/ hmbci-2016-0023

Conflict of Interest Statement: The authors declare that the research was conducted in the absence of any commercial or financial relationships that could be construed as a potential conflict of interest.

Copyright (® 2018 Toloza, Mantilla-Rivas, Pérez-Matos, Ricardo-Silgado, MoralesAlvarez, Pinzón-Cortés, Pérez-Mayorga, Arévalo-Garcia, Tolosa-González and Mendivil. This is an open-access article distributed under the terms of the Creative Commons Attribution License (CC BY). The use, distribution or reproduction in other forums is permitted, provided the original author(s) and the copyright owner are credited and that the original publication in this journal is cited, in accordance with accepted academic practice. No use, distribution or reproduction is permitted which does not comply with these terms. 